\title{
Apparatus for dimensional characterization of fused silica fibers for the suspensions of advanced gravitational wave detectors
}

\author{
A. Cumming, ${ }^{1, a)}$ R. Jones, ${ }^{1}$ M. Barton, ${ }^{2}$ G. Cagnoli, ${ }^{3}$ C. A. Cantley, ${ }^{1}$ D. R. M. Crooks, ${ }^{1}$ \\ G. D. Hammond,${ }^{1}$ A. Heptonstall, ${ }^{2}$ J. Hough, ${ }^{1}$ S. Rowan, ${ }^{1}$ and K. A. Strain ${ }^{1}$ \\ ${ }^{1}$ SUPA Institute for Gravitational Research, Department of Physics and Astronomy, University of Glasgow, \\ Glasgow G12 8QQ, United Kingdom \\ ${ }^{2}$ LIGO Laboratory, California Institute of Technology, Pasadena, California 91125, USA \\ ${ }^{3}$ University of Texas at Brownsville, Department of Physics and Astronomy, Brownsville, Texas 78526, USA
}

(Received 12 January 2011; accepted 30 March 2011; published online 21 April 2011)

\begin{abstract}
Detection of gravitational waves from astrophysical sources remains one of the most challenging problems faced by experimental physicists. A significant limit to the sensitivity of future longbaseline interferometric gravitational wave detectors is thermal displacement noise of the test mass mirrors and their suspensions. Suspension thermal noise results from mechanical dissipation in the fused silica suspension fibers suspending the test mass mirrors and is therefore an important noise source at operating frequencies between $\sim 10$ and $30 \mathrm{~Hz}$. This dissipation occurs due to a combination of thermoelastic damping, surface and bulk losses. Its effects can be reduced by optimizing the thermoelastic and surface loss, and these parameters are a function of the cross sectional dimensions of the fiber along its length. This paper presents a new apparatus capable of high resolution measurements of the cross sectional dimensions of suspension fibers of both rectangular and circular cross section, suitable for use in advanced detector mirror suspensions. () 2011 American Institute of Physics. [doi:10.1063/1.3581228]
\end{abstract}

\section{INTRODUCTION}

Long baseline interferometers are used to search for the effects of gravitational waves from astronomical sources by sensing the relative displacements of mirrors suspended as pendulums at the ends of perpendicular interferometer arms. Typical midrange detection band frequencies are $\sim 10-500$ $\mathrm{Hz}$, where an important limit to displacement sensitivities is set by thermal motion of the test masses and their suspension elements. Pendulum suspensions are used to isolate the test mass mirrors from the effects of seismic noise. ${ }^{1}$ Mechanical dissipation in these suspensions, together with dissipation in the test mass mirrors gives rise to thermal displacement noise ${ }^{2}$ the magnitude of which can be calculated through application of the fluctuation-dissipation theorem. ${ }^{3,4}$ The thermal noise in the suspension elements is most significant in the band between 10 and $30 \mathrm{~Hz} .^{5}$ By using materials with very low mechanical dissipation, the suspension thermal noise at these frequencies can be reduced. The current material of choice is fused silica, which has been demonstrated to have suitably low loss, permitting a significant reduction in the thermal noise contributed from the pendulum mode of the suspension as compared to use of metal wire suspensions. ${ }^{6-13}$

Currently, several first generation interferometric gravitational wave detectors worldwide have completed data taking runs operating at, or close to, their design sensitivities, including the (LIGO) network, ${ }^{14}$ the Virgo detector, ${ }^{15,16}$ the GEO600 detector, ${ }^{17,18}$ and the TAMA instrument. ${ }^{19}$ GEO600 was the first to employ ultra low loss quasimonolithic fused silica suspensions in its design. ${ }^{20}$ It employs three stage

\footnotetext{
a) Author to whom correspondence should be addressed. Electronic mail: a.cumming@physics.gla.ac.uk. Telephone: 0141330 8237. Fax: 0141330 6833.
}

mirror suspensions, and was the first detector where the final stage test masses are suspended using four fused silica fibers which are welded to small fused silica attachment pieces ("ears"). These ears are attached to the sides of the test mass by hydroxide-catalysis bonds. ${ }^{21,22}$ The LIGO and Virgo detectors use fused silica mirrors which are suspended with steel suspension wires that have mechanical loss factors several orders of magnitude greater than fused silica. $^{12,23}$

Forthcoming upgrades of the LIGO network to the "Advanced LIGO" system will introduce quasimonolithic silica suspensions similar to those used in GEO600, but on a larger scale, with $40 \mathrm{~kg}$ masses suspended from circular cross section fused silica fibers as shown in Fig. $1 .{ }^{24}$ Silica suspensions are also being used in ongoing upgrades to Virgo. ${ }^{25}$ Rectangular cross section fibers were also considered for use in advanced detectors and future detectors may utilize rectangular cross section fibers. ${ }^{8,9,13,26}$

Previous analyses of fused silica fibers used in suspensions ${ }^{27-32}$ have shown that the dimensions of suspension fibers, and in particular variations in the cross section of the fibers along their length, can strongly influence the dissipation associated with the pendulum mode of a suspension. Therefore accurate dimensional characterization of suspension fibers is required to allow the prediction of suspension thermal noise. Additionally, for the Advanced LIGO detector system, suspension fibers are required to have a tolerance of $\pm 2.8 \%$ in vertical stiffness set by the maximum $20 \mathrm{mrad}$ range of the mirror steering actuators. ${ }^{33,34}$ This yields a required cross sectional dimensional tolerance of $\pm 1.9 \%$ in fiber diameter along the fiber length ${ }^{33}$ - therefore any dimensional characterization device is required to be able to measure fiber dimensions to within this tolerance. This paper describes the 


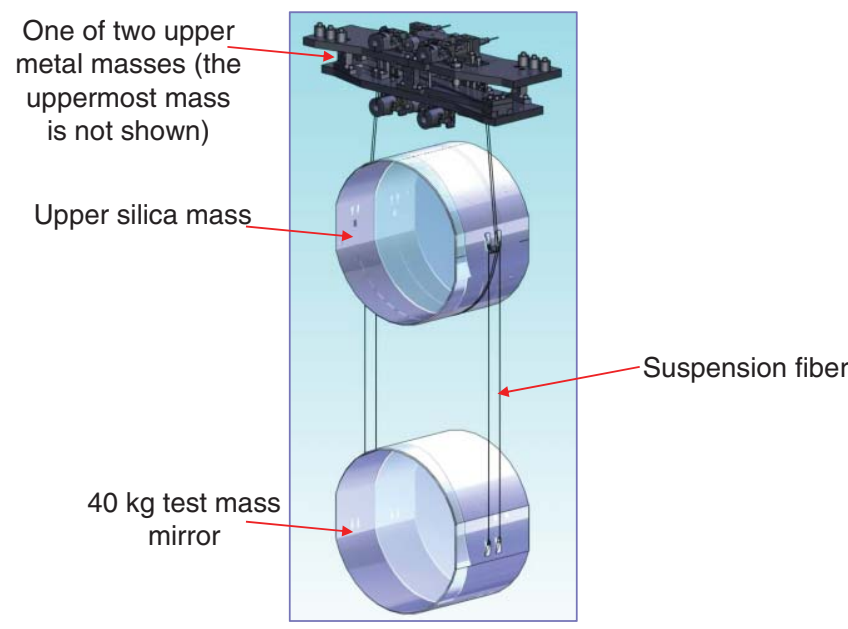

FIG. 1. (Color online) Advanced LIGO lower suspension stage showing the monolithic portion and one of two upper metal masses.

design and function of a new apparatus developed to fulfill this requirement.

\section{DISSIPATION IN MIRROR SUSPENSION FIBERS}

Dissipation at some angular frequency, $\omega$, is characterized by the mechanical loss, $\phi(\omega)$ of a material. ${ }^{2}$ The mechanical loss of a suspension fiber, $\phi_{\text {fiber }}(\omega)$, can typically be expressed as the sum of three loss components-a frequency dependent loss $\phi_{\text {bulk }}(\omega)$ associated with the bulk material making up the fiber, ${ }^{35}$ a component from loss arising at the fiber surface $\phi_{\text {surface }}$ often assumed to be frequency independent, ${ }^{36}$ and a frequency dependent thermoelastic loss component, $\phi_{\text {thermoelastic }}(\omega){ }^{37}$ The total loss is given by $^{30}$

$$
\phi_{\text {fiber }}(\omega)=\phi_{\text {surface }}+\phi_{\text {bulk }}(\omega)+\phi_{\text {thermoelastic }}(\omega) \text {. }
$$

For thin suspension fibers where the surface to volume ratio is very large $\phi_{\text {bulk }}(\omega)$ is negligible. The dominant loss contributions are therefore $\phi_{\text {surface }}$ and $\phi_{\text {thermoelastic }}(\omega)$. For a circular cross section fiber the thermoelastic loss is given by ${ }^{32}$

$$
\phi_{\text {thermoelastic }}(\omega)=\frac{Y T}{\rho C}\left(\alpha-\sigma_{o} \frac{\beta}{Y}\right)^{2}\left(\frac{\omega \tau}{1+(\omega \tau)^{2}}\right) .
$$

with

$$
\tau=\frac{1}{4.32 \pi} \frac{\rho C d^{2}}{\kappa}
$$

with $\tau$ being the characteristic time for heat to travel from one side of the fiber to the other, $Y$ is the Young's modulus of the fiber, $C$ is the specific heat capacity of the material, $\kappa$ is its thermal conductivity, $\rho$ is its density, $\alpha$ is the coefficient of linear thermal expansion, $\sigma_{o}$ is the static stress in the fiber due to the suspended load, $\beta=\frac{1}{Y} \frac{d Y}{d T}$ is the thermal elastic coefficient, $T$ is the temperature, and $d$ is the diameter of the fiber.

Fused silica has a $\beta$ value which is positive, ${ }^{32,38}$ meaning that thermoelastic loss can be reduced by application of an

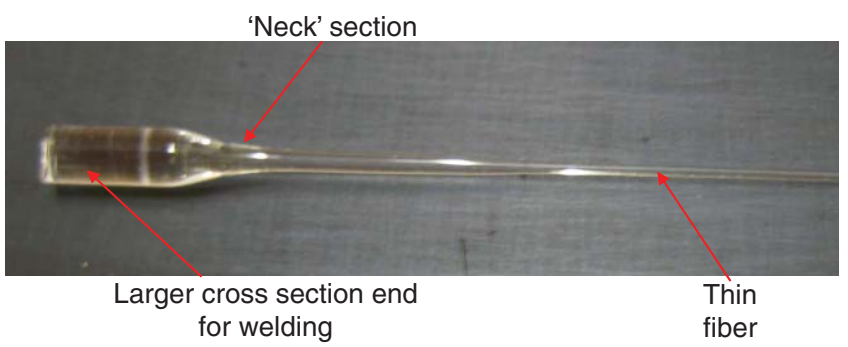

FIG. 2. (Color online) Prototype Advanced LIGO fiber end.

appropriate static stress, and in principle nulled entirely when

$$
\sigma_{o}=\frac{\alpha Y}{\beta}
$$

For a given suspension payload there is therefore an optimal fiber cross section for minimizing pendulum mode loss by nulling thermoelastic noise. Advanced LIGO is one of the advanced detectors whose suspensions aim to use this phenomenon to minimize mechanical dissipation by use of "dumbbell" fibers with dimensions of $800 \mu \mathrm{m}$ at the fiber ends where most bending occurs. ${ }^{30,39}$ This $800 \mu \mathrm{m}$ section allows cancellation of thermoelastic noise for a nominal static loading of $97.1 \mathrm{~N}$, per fiber. The central section of the fiber is designed to be thinner at $400 \mu \mathrm{m}$ diameter to keep the vertical frequency of the suspension lower than $10 \mathrm{~Hz}$ and therefore outwith the detection band; and raise the violin mode frequencies to $\sim 500 \mathrm{~Hz}^{24}$

Current fiber production methods involve drawing fibers from heated fused silica rods or slides of a larger cross section than the resulting fiber. ${ }^{40}$ The fiber ends therefore have a tapered "neck" section as shown in Fig. 2, increasing in dimension to a larger cross section (typically a few millimeters in diameter) which is more suitable for welding to the silica ears bonded to the sides of the test mass. Typically, this neck is between 5 and $15 \mathrm{~mm}$ in length. ${ }^{40}$

As described in Refs. 27 and 30 some elastic energy can be stored in this neck region in a test mass suspension meaning that some thermoelastic loss will occur in this area of the fiber since the dimensions differ from that required for nulling thermoelastic loss. Quantifying this loss is important for characterizing detector performance. ${ }^{30}$ Hence, accurate measurements of the dimensions of a fiber are crucial for calculating performance and providing feedback to optimize the production process. ${ }^{30}$

The effect of surface loss on suspension performance is also dependent on the dimensions of the fiber used, and for a circular fiber the contribution of surface loss to the total fiber loss is given by ${ }^{13}$

$$
\phi_{\text {surface fiber }}=\frac{8 h \phi_{s}}{d}
$$

where $h \phi_{s}$ is the product of the mechanical loss of the surface of the material, $\phi_{s}$, and depth, $h$, over which surface loss mechanisms are believed to occur. Again, knowledge of the fiber dimensions is necessary to quantify accurately the contribution of surface loss. 
The mechanical loss of a full pendulum suspension, $\phi_{\text {pendulum }}$ can be shown to be ${ }^{41}$

$$
\phi_{\text {pendulum }}(\omega) \approx \phi_{\text {fiber }}(\omega) \frac{k_{\text {fiber }}}{k_{\text {gravity }}},
$$

where $k_{\text {fiber }}$ is the suspension fiber spring constant due to the material elasticity, and $k_{\text {gravity }}$ is the effective spring constant arising from gravity. The ratio of elastic and gravitational spring constants is the pendulum dilution factor, $D$, and is also dependent on the fiber cross sectional dimensions, ${ }^{41}$

$$
D \approx \frac{k_{\text {gravity }}}{k_{\text {fiber }}}=\frac{16 m g l}{\sqrt{F Y \pi d^{4}}}
$$

where $m$ is the pendulum mass, $l$ is the pendulum length, and $F$ is the tension in the pendulum suspension fiber.

It is therefore clear that all aspects of the suspension thermal noise depend on the fiber cross sectional dimensions. Quantifying these dimensions is crucial for characterizing these aspects of the fiber's mechanical dissipation, allowing optimization of the fibers to be used in advanced and future gravitational wave detectors.

\section{DESCRIPTION OF HARDWARE}

The fiber dimensional characterization machine comprises an imaging head mounted on a motorized traveling carriage and ball screw tower, with clamping fixtures top and bottom to hold the fiber as shown in Fig. 3.

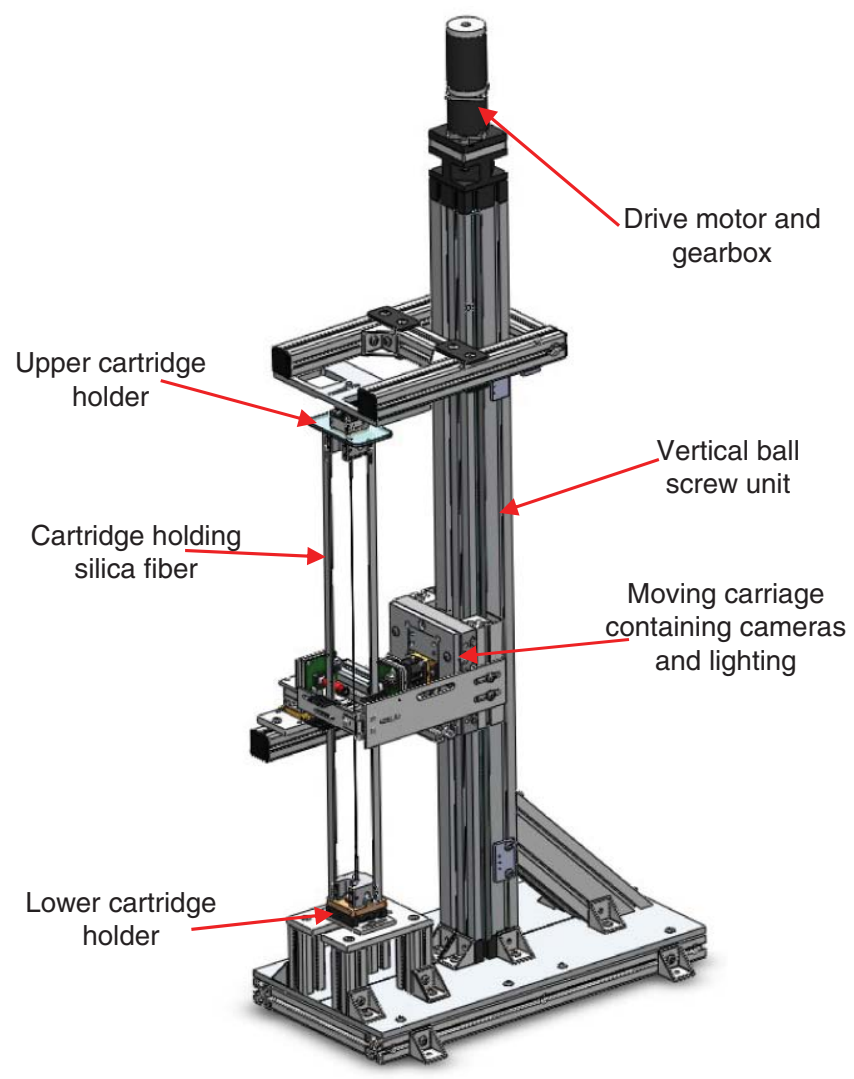

FIG. 3. (Color online) Rendering of the dimensional characterization machine showing main components.
Mounted on the carriage are two identical orthogonal cameras (Unibrain Fire-i Digital Board Camera, Monochrome, part \#2057), ${ }^{42}$ with lenses chosen such that one camera has high linear magnification of 6.15 , and one has low linear magnification of 1.75 . The different magnifications were chosen to obtain the optimum resolution in the measurement of the different regions of the fiber. The low magnification camera is able to measure the dimensions of the thicker ends of the necks and the thinner central section can be studied by the higher magnification camera. This configuration also permits the measurement of rectangular cross section fibers more easily. The low magnification camera is mounted on the bed of the carriage with the higher magnification camera perpendicular to this as shown in more detail in Fig. 4. Additional depth of field is also gained on the high magnification camera by use of an aperture size of $\sim 0.75 \mathrm{~mm}$, giving f-number of $f / 11$. This ensures there is sufficient depth of field that both edges of the fiber are in focus for this camera. The pixel resolution of each camera is $640 \times 480$, and with the two different magnification lenses the measurement resolution attained by the two cameras are $9.6 \mu \mathrm{m}$ per pixel for the low magnification camera, and $1.6 \mu \mathrm{m}$ per pixel for the high magnification camera.

Diffuse backlighting is provided using high intensity $630 \mathrm{~nm}$ red "Lumiled" Luxeon Star $350 \mathrm{~mA}$ light-emitting diodes (LEDs) ${ }^{43}$ whose lens is removed and replaced with a teflon diffuser to give a diffuse background light. These LED backlights are also mounted on the carriage so they travel with the cameras. One backlight is hinged to allow the fiber to be easily removed.

Travel of the carriage along the fiber is measured using a magnetic linear position encoder with $0.1 \mathrm{~mm}$ resolution [Siko MSK320 (Ref. 44)] mounted to the rear of the carriage.

Fibers are produced by a $\mathrm{CO}_{2}$ laser based pulling machine.$^{40}$ Once produced, the fibers need to be handled with care, and the section of the fiber that is used in the detector suspension must not be touched. Any contact with the fiber risks introducing microcracks to the fiber surface which can compromise strength. ${ }^{45-47}$ Therefore a robust method for transportation and handling of fibers between laser pulling machine and dimensional characterization machine was required. To meet this demand, a "cartridge" system that interfaces with both machines was developed as shown in Fig. 5. Cylindrical metal holsters are attached to the ends of the fibers before pulling with an epoxy adhesive to allow the fiber to be handled latterly without the cartridge. These attach into metal cube clamps, which interface with the pulling machine and are braced with angle section bars to form the rigid cartridge that holds the fiber in light tension after pulling. Use of different length bracing bars allows accommodation of fibers of any length.

Cartridges can be transferred between pulling machine and dimensional characterization machine, with the ends of the clamps engaging into circular bearings on the dimensional characterization machine as shown in Fig. 6. The clamping fixtures at the top and bottom also have adjustability built in to allow coarse positioning of the cartridge relative to the cameras. Fine positioning and focus of the cameras is 


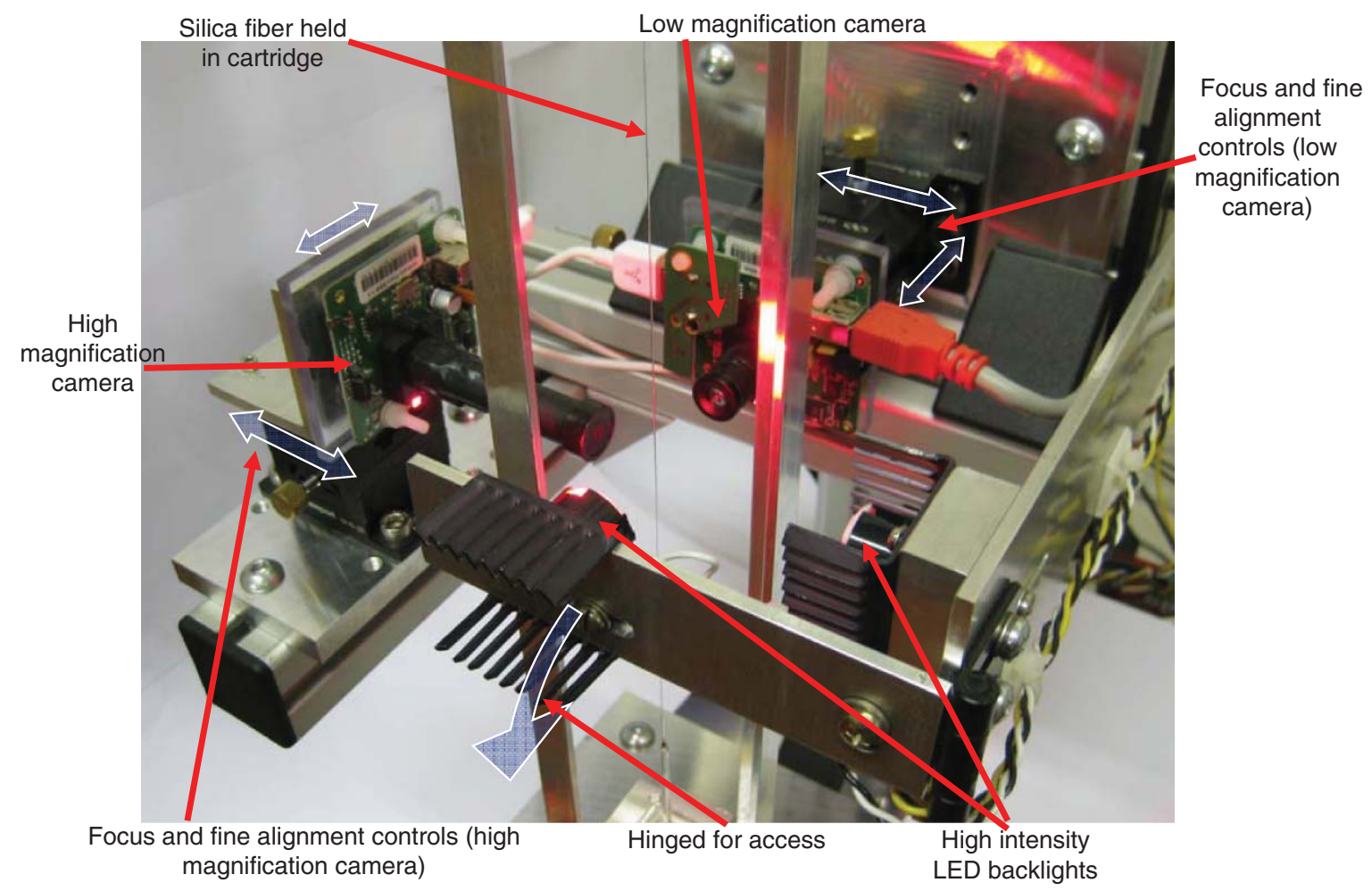

FIG. 4. (Color online) Dimensional characterization machine imaging head showing orthogonal camera setup and backlighting configuration.

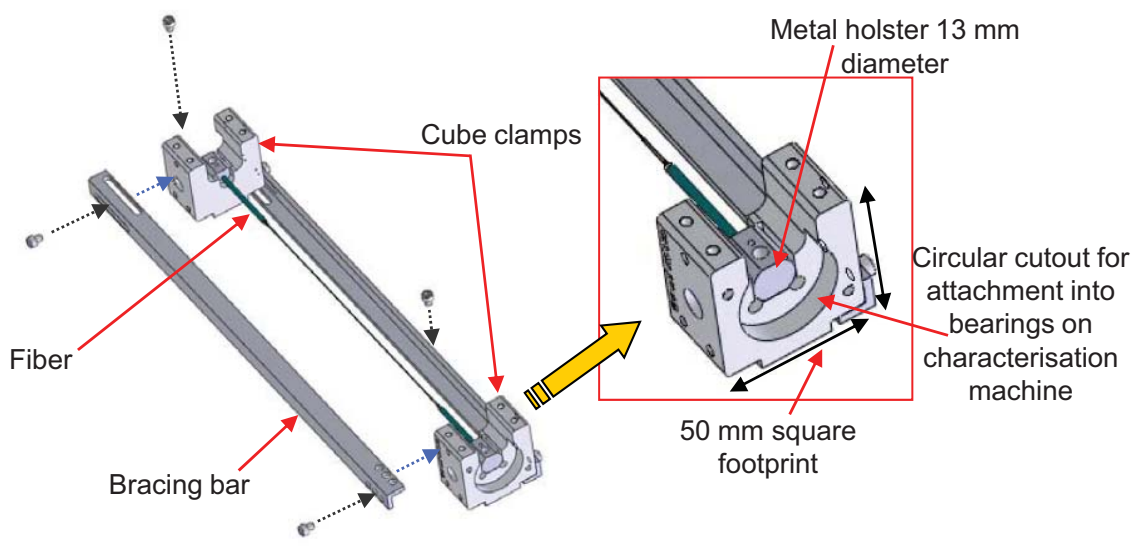

FIG. 5. (Color online) Fiber cartridge system used to transport fibers between pulling and characterization machines (shortened length version shown for clarity).

(a)

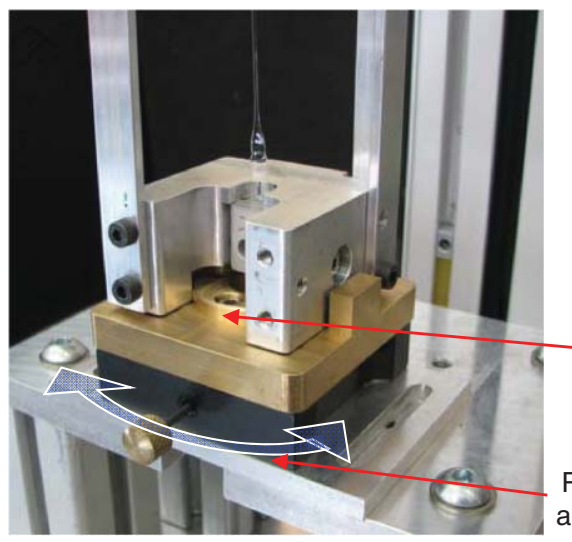
adjustment

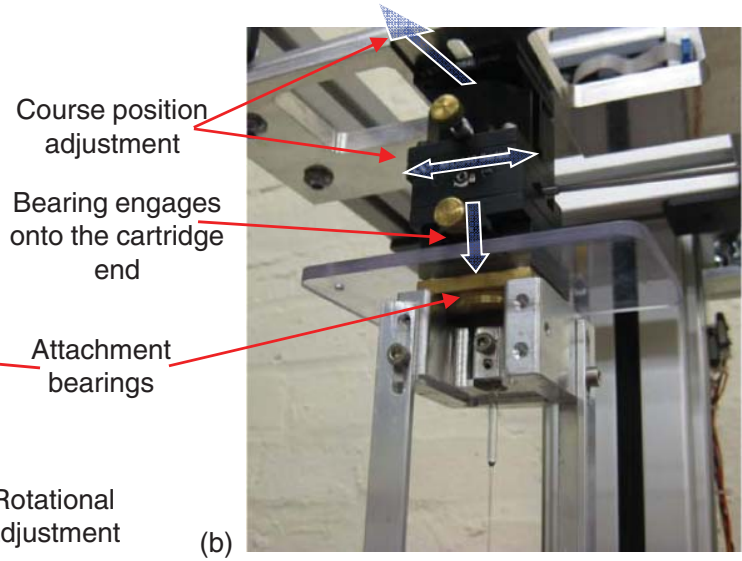

(b)

FIG. 6. (Color online) Cartridge clamp interfaces at (a) bottom (b) top of dimensional characterization machine. 


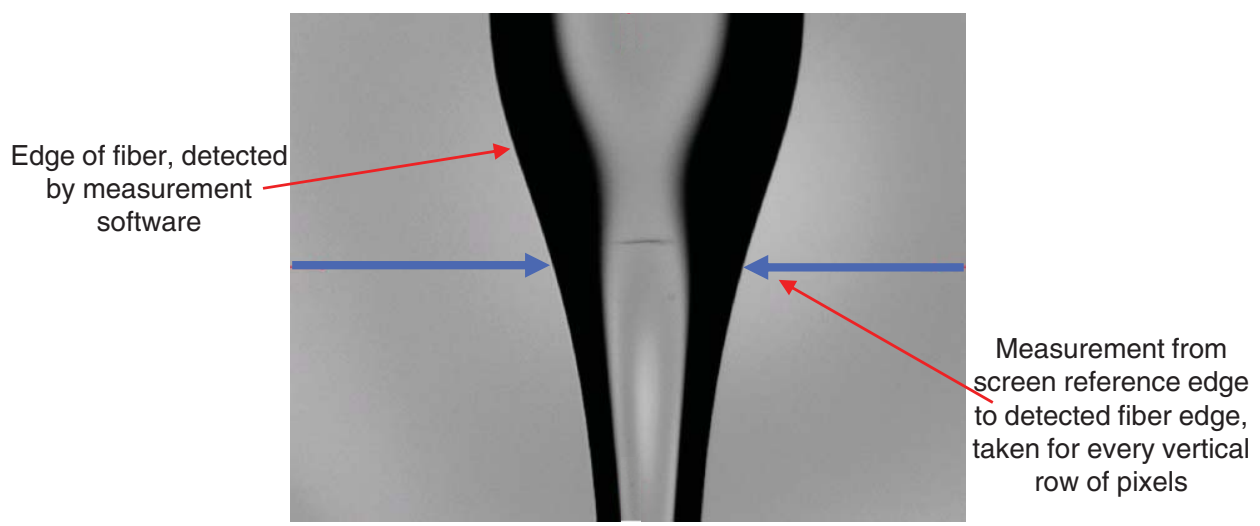

FIG. 7. (Color online) Typical observed image of a circular cross section fiber neck. The central lighter region occurs due to backlight being transmitted through the fiber.

accomplished using translation stages mounted on the bases of the cameras themselves as previously discussed and shown in Fig. 4.

\section{MEASUREMENT SOFTWARE}

A custom National Instruments LABVIEW program is used to control the machine and perform the measurements, ${ }^{29,48}$ via a LabJack U12 USB data acquisition card. ${ }^{49}$ The program uses contrast edge detection to determine the position of the fiber's outside edges as shown in Fig. 7. The program employs two modes of operation; the first mode is used for the fiber neck regions, where an edge detection measurement is performed on every row of pixels of the image. The camera head moves up by less than the field of view of the camera automatically once measurements are taken on one image and repeats this process, giving measurements along the fiber neck length. These measurements are then automatically stitched together by the program with any overlap taken into account, and give both the width and thickness (from the independent orthogonal cameras) along the length of the neck region.

Along the central thin section of the fiber a second mode of operation is employed where measurements are taken at user defined intervals along the ribbon length, typically every 5 to $10 \mathrm{~mm}$. This permits the dataset for a fiber to be kept to a manageable size. Averaging over multiple pixels for a short length of fiber can also be employed (typically $\sim 20$ pixels). No interference between the orthogonal light sources occurs, so in both modes of operation the program operates simultaneously on the images captured from the two cameras, with the measurement, calculation and recording taking less than $1 \mathrm{~s}$ per image. Measurement of a full $60 \mathrm{~cm}$ fiber typically takes 15-20 min, the majority of this time being taken up with focusing and movement between measurement points.

A typical dimension profile of a suspension fiber suitable for use in advanced gravitational wave detectors is shown in Fig. 8.

In addition, the neck region scan also measures the position of both edges of the fiber, referenced from the edge of

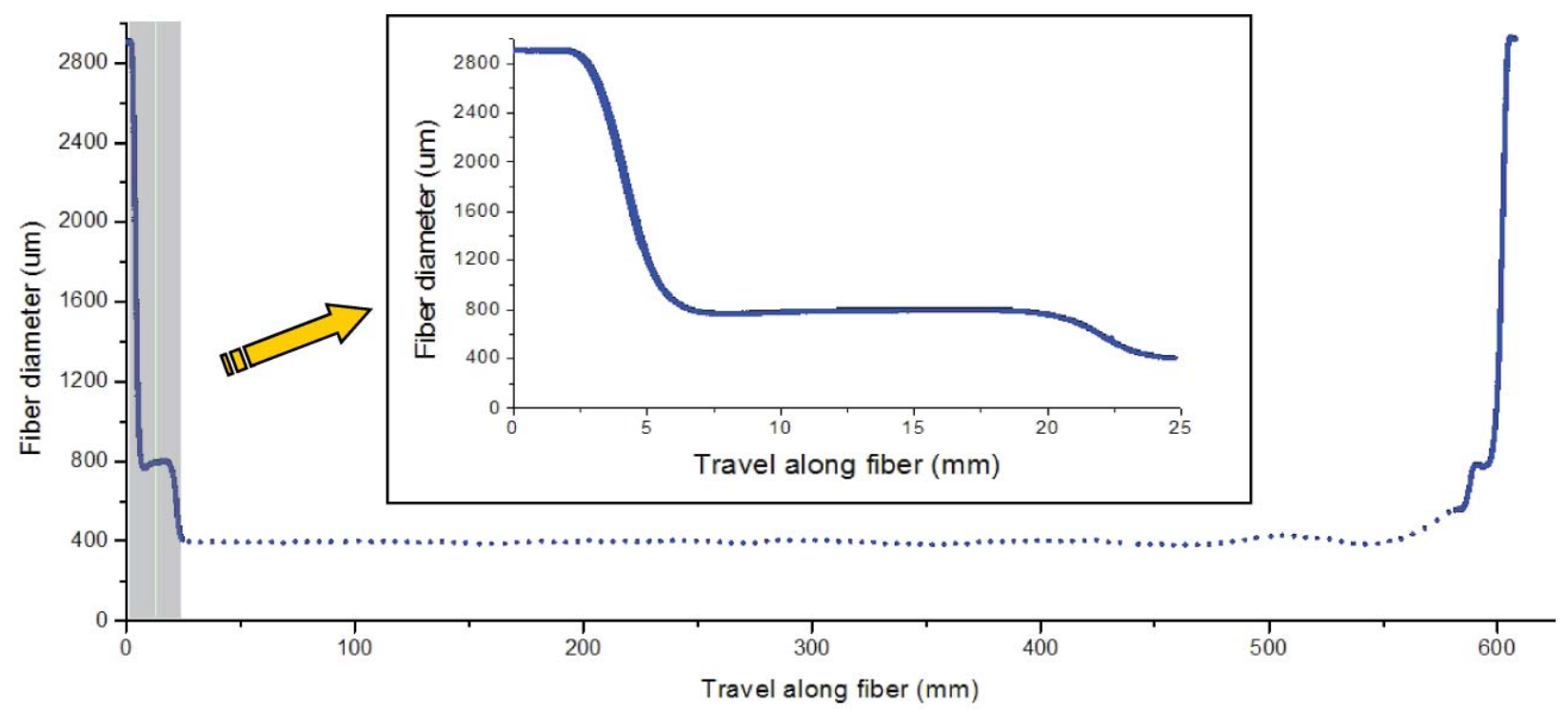

FIG. 8. (Color online) Typical full fiber profile as obtained directly from the dimensional characterization machine, of a suspension fiber suitable for use in advanced gravitational wave detectors, pulled using the $\mathrm{CO}_{2}$ laser pulling machine. ${ }^{40}$ The plot shows high resolution measurements taken in the neck region of the fiber and lower resolution measurements taken in the midsection. Inset plot shows in more detail the high resolution scan of shaded area containing the first neck region of the fiber, with $800 \mu \mathrm{m}$ dumbbell section to minimize thermoelastic loss. 


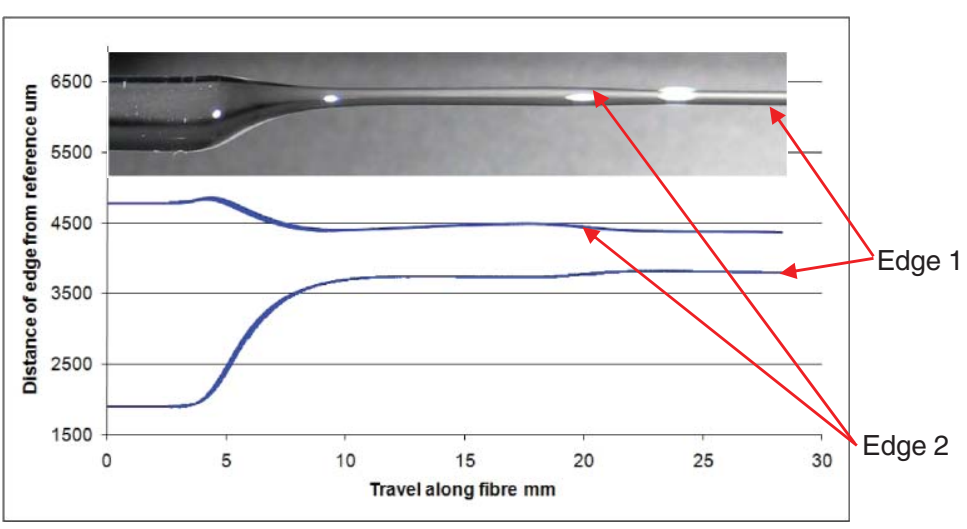

FIG. 9. (Color online) Scan of the edge position of a fiber end for illustrative purposes, showing the effect of a slight misalignment that has occurred during production.

the image obtained from the cameras. This dimensioned shape profile gives a method of quantifying any axial misalignment of the fiber neck relative to the larger cross section weld region, giving information that can be used to make quantified adjustments of the fiber pulling process. A neck edge scan of a misaligned fiber is shown in Fig. 9.

Errors in the measurements are small compared to the dimensions being measured. A systematic error can be introduced in the calibration of the machine with the limitation coming from the accuracy in knowledge of the dimension of the calibrating device. Calibration is therefore undertaken with slip gauges, ensuring that it is not the limiting factor. Typically these are of dimension $1000 \pm 1 \mu \mathrm{m}$ for the low magnification camera, therefore meaning the systematic error due to the accuracy of the calibration device is of the order of $1 \mu \mathrm{m}(0.1 \%)$. Additionally, slip gauges showed that the geometric distortion due to the quality of the lenses was less than $0.4 \%$.

The spread in measurements is seen due to focusing is observed to be $0.25 \%$ in fiber diameter for the low magnification camera and $1 \%$ in the fiber diameter for the high magnification camera if repeated measurement on the same fiber is undertaken. This random error occurs due to small variations in the edge detection measurement from small ambient lighting fluctuations.

Typically the measurements from the high magnification camera are taken for the thin section of the fiber, and the low magnification camera measurements used for the thicker fiber ends where a greater field of view is required. For a $400 \mu \mathrm{m}$ diameter fiber, quadrature sum of the errors from the high magnification camera yields a total error of $1.15 \%$. For an $800 \mu \mathrm{m}$ fiber section, the quadrature sum of the errors from the low magnification camera is $1.29 \%$. Therefore the apparatus allows fibers outwith the $1.9 \%$ diameter requirement for Advanced LIGO to be identified from the resultant profiles. Should additional accuracy be required, repeated measurement runs along a given fiber can be conducted and measurements averaged; though with the observed errors present, this has not proved necessary in practice. Comparison of violin mode frequencies of LIGO fibers with FEA models made using dimensional data from the characterization machine have demonstrated excellent agreement, to within $0.4 \%,{ }^{50}$ giving additional verification of the accuracy of the apparatus.
The ability to profile fibers using this dimensional characterization machine will permit dimensions of real fibers to be incorporated into finite element models of mirror suspensions (as studied for analytical shapes in Ref. 30) allowing suspension thermal noise calculations to be undertaken for the first time using real suspension elements, allowing prediction of the performance of these elements in future gravitational wave detector mirror suspensions.

\section{CONCLUSIONS}

Suspension thermal noise in the advanced detectors will be directly dependent on the dimensions of the fibers used to suspend the mirrors. Accurately quantified fused silica fiber dimension profiles are therefore of great importance for attaining improved thermal noise performance at low frequency in advanced interferometric gravitational wave detectors. An apparatus suitable for measurement of either rectangular or circular cross section fibers has been demonstrated in this paper. A duplicate apparatus was installed at the LIGO LASTI test facility at MIT and was used to check the dimensional quality of fibers used in constructing the first Advanced LIGO noise prototype suspension. This has subsequently been relocated to the LIGO Hanford Washington detector site, in readiness for continued use in checking the quality of suspension fibers produced for suspensions that will be used in upgrade of the LIGO detector network to Advanced LIGO.

\section{ACKNOWLEDGMENTS}

The authors would like to thank Colin Craig and Stephen Craig for their work toward the construction of the machine. We would like to thank our colleagues in the GEO600 project, the LSC-Virgo collaboration, and within SUPA for their interest in this work. We are grateful for the financial support provided by Science and Technology Facilities Council (STFC), the Scottish Funding Council (SFC), the Royal Society, the Wolfson Foundation, and the University of Glasgow in the UK. We would also like to thank the National Science Foundation (NSF) in the USA (Award Nos. PHY-05 02641 and PHY-07 57896). LIGO was constructed by the California Institute of Technology and Massachusetts Institute of Technology with funding from the National Science Foundation 
(NSF) and operates under cooperative agreement PHY0107417. We would like to thank our colleagues in the LSC and Virgo collaborations and within SUPA for their interest in this work. This paper has LIGO document number P1000178.

${ }^{1}$ P. R. Saulson, Fundamentals of Interferometric Gravitational Wave Detectors (World Scientific, London, 1994).

${ }^{2}$ P. R. Saulson, Phys. Rev. D 42(8), 2437 (1990).

${ }^{3}$ H. B. Callen and T. A. Welton, Phys. Rev. 83(1), 34 (1951).

${ }^{4}$ H. B. Callen and R. F. Greene, Phys. Rev. 86(5), 702 (1952).

${ }^{5}$ S. Rowan and J. Hough, Living Rev. Relativ. 3, 3 (2000).

${ }^{6}$ V. B. Braginsky, V. P. Mitrofanov, and K. V. Tokmakov, Phys. Lett. A 186, 18 (1994).

${ }^{7}$ V. B. Braginsky, V. P. Mitrofanov, and K. V. Tokmakov, Phys. Lett. A 218 , 164 (1996)

${ }^{8}$ S. Rowan, R. Hutchins, A. McLaren, N. A. Robertson, S. M. Twyford, and J. Hough, Phys. Lett. A 227, 153 (1997).

${ }^{9}$ A. Heptonstall, "Characterisation of mechanical loss in fused silica ribbons for use in gravitational wave detector suspensions," Ph.D. dissertation (The University of Glasgow, 2004).

${ }^{10}$ V. B. Braginsky, Systems with Small Dissipation (University of Chicago, Chicago, 1985).

${ }^{11}$ Y. L. Huang and P. R. Saulson, Rev. Sci. Instrum. 69, 544 (1998).

${ }^{12}$ G. Cagnoli, L. Gammaitoni, J. Kovalik, F. Marchesoni, and M. Punturo, Phys. Lett. A 255, 230 (1999).

${ }^{13}$ A. Gretarsson, G. M. Harry, S. D. Penn, P. R. Saulson, W. J. Startin, S. Rowan, G. Cagnoli, and J. Hough, Phys. Lett. A 270, 108 (2000).

${ }^{14}$ R. Frey, LIGO: Status and Recent Results, C2CR07 proceedings, LIGO Document P070079-01-Z, 2007.

${ }^{15}$ C. Bradashia, R. D. Fabbro, A. D. Virgilio, A. Giazotto, H. Kautzky, V. Montelatici, D. Passuello, A. Brillet, O. Cregut, P. Hello, C. N. Man, P. T. Manh, A. Marraud, D. Shoemaker, and J. Y. Vinet, Nucl. Instrum. Methods Phys. Res. A289 518 (1990).

${ }^{16} \mathrm{http}: / /$ wwwcascina.virgo.infn.it/MonitoringWeb/General/archive/VSR1/ Sensitivity.jpg for recent strain sensitivity curves for Virgo.

${ }^{17}$ B. Willke, P. Aufmuth, C. Aulbert, S. Babak, R. Balasubramanian, B. W. Barr, S. Berukoff, S. Bose, G. Cagnoli, M. M. Casey, D. Churches, and D. Clubley, Class. Quantum Grav. 19, 1377 (2002).

${ }^{18}$ See http://www.geo600.uni-hannover.de/geocurves/files/theoretical/all_ 250_v4.png for recent strain sensitivity curves for GEO600.

${ }^{19}$ R. Takahashi, Class. Quantum Grav. 21, S403 (2004).

${ }^{20}$ M. V. Plissi, K. A. Strain, C. I. Torrie, and N. A. Robertson, Rev. Sci. Instrum. 69, 3055 (1998).

${ }^{21}$ S. Rowan, S. M. Twyford, J. Hough, D. H. Gwo, and R. Route, Phys. Lett. A 246, 471 (1998).

${ }^{22}$ L. Cunningham, P. G. Murray, A. Cumming, E. J. Elliffe, G. D. Hammond, K. Haughian, J. Hough, M. Hendry, R. Jones, I. W. Martin, S. Reid, S. Rowan, J. Scott, K. A. Strain, K. Tokmakov, C. Torrie, and A. A. Veggel, Phys. Lett. A 374, 3993 (2010).

${ }^{23}$ J. Kovalik and P. R. Saulson, Rev. Sci. Instrum. 64, 2942 (1993)

${ }^{24}$ N. A. Robertson, G. Cagnoli, D. R. M. Crooks, E. Elliffe, J. E. Faller, P. Fritschel, S. Goßler, A. Grant, A. Heptonstall, J. Hough, H. Luck, R. Mittleman, and M. Perreur-Lloyd, Class. Quantum Grav. 19, 4043 (2002).
${ }^{25}$ P. Amico, L. Bosi, L. Carbone, L. Gammaitoni, M. Punturo, F. Travasso, and H. Vocca, Class. Quantum Grav. 19, 1669 (2002).

${ }^{26}$ S. Reid, G. Cagnoli, D. R. M. Crooks, J. Hough, P. Murray, S. Rowan, M. M. Fejer, R. Route, and S. Zappe, Phys. Lett. A 351, 205 (2005).

${ }^{27}$ P. Willems and M. Thattai, Phys. Lett. A 253, 16 (1999).

${ }^{28}$ P. Willems, Phys. Lett. A 300, 162 (2002).

${ }^{29} \mathrm{~A}$. Cumming, "Aspects of mirrors and suspensions for advanced gravitational wave detectors," Ph.D. dissertation (The University of Glasgow, 2008)

${ }^{30}$ A. Cumming, A. Heptonstall, R. Kumar, W. Cunningham, C. Torrie, M. Barton, K. A. Strain, J. Hough, and S. Rowan, Class. Quantum Grav. 26(21), 215012 (2009).

${ }^{31}$ Kumar R., "Finite element analysis of suspension elements for gravitational wave detectors," M.S. thesis (The University of Glasgow, 2008).

${ }^{32}$ G. Cagnoli and P. Willems, Phys. Rev. B 65, 174111 (2002).

${ }^{33} \mathrm{G}$. Cagnoli, Ribbon tolerances and alignment requirements for Advanced LIGO optics, LIGO Document T050212, 2005.

${ }^{34} \mathrm{~N}$. Robertson, M. Barton, and J. Greenhalgh, Cross-coupling in quadruple pendulum suspension, LIGO Document T040143, 2004.

${ }^{35}$ S. D. Penn, A. Ageev, D. Busby, G. M. Harry, A. M. Gretarsson, K. Numata, and P. Willems, Phys. Lett. A 352, 3 (2006).

${ }^{36}$ A. Gretarsson and G. M. Harry, Rev. Sci. Instrum. 70(10), 4081 (1999).

${ }^{37}$ A. Nowick and B. Berry, Anelastic Relaxation in Crystalline Solids (Academic Press, London, 1972).

${ }^{38}$ P. Willems, V. Sannibale, J. Weel, and V. Mitrofanov, Phys. Lett. A 297, 37 (2002).

${ }^{39}$ M. Barton, A. Heptonstall, C. Craig, A. Cumming, L. Cunningham, G. Hammond, K. Haughian, J. Hough, R. Jones, R. Kumar, N. Robertson, S. Rowan, K. Strain, K. Tokmakov, and C. Torrie, Proposal for baseline change from ribbons to fibers in AdvLIGO test mass suspension monolithic stage, LIGO Document T080091, 2008.

${ }^{40}$ A. Heptonstall, M. A. Barton, A. Bell, G. Cagnoli, C. A. Cantley, D. R. M. Crooks, A. Cumming, A. Grant, G. D. Hammond, G. M. Harry, J. Hough, R. Jones, D. Kelley, R. Kumar, I. W. Martin, N. A. Robertson, S. Rowan, K. A. Strain, K. Tokmakov, and A. A. Veggel, Rev. Sci. Instrum. 82, 011301 (2011).

${ }^{41}$ G. Cagnoli, J. Hough, D. DeBra, M. M. Fejer, E. Gustafson, S. Rowan, and V. Mitrofanov, Phys. Lett. A 272, 39 (2000).

${ }^{42}$ See http://www.unibrain.com/Products/VisionImg/Fire_i_BC.htm for technical details of Unibrain Fire-i board level webcameras.

${ }^{43}$ See http://www.lumileds.com/products/line.cfm?lineId=1 for technical details of Luxeon Star Lumiled high intensity LEDs.

${ }^{44}$ See http://www.siko.de/en/produkte/magline/sensors/details/msk320/ for technical details of Siko MSK320 linear position encoder.

${ }^{45}$ R. K. Brow, N. P. Lower, and A. J. Lang, in Proceedings of the 7th International Otto Schott Colloquium, July 7-11, 2002; Glastech. Ber. Glass Sci. Technol. 75 C2 133-138 (2002).

${ }^{46}$ B. Proctor and I. Whitney, Proc. R. Soc. London, Ser. A 297, 1451 (1967)

${ }^{47}$ C. Kurkjian, J. Krause, and M. Matthewson, J. Lightwave Technol. 7, 1360 (1989).

${ }^{48}$ A. Cumming, LIGO Document T100024, 2010.

${ }^{49}$ See http://labjack.com/u12 for technical details of Labjack U12 data acquisition device.

${ }^{50}$ A. Cumming, LIGO Document T0900299, 2009. 